The Allied Health SChOlar

\title{
Neonatal Occupational Therapy in Australia: A Survey and Recommendations for Developing Guidelines for Clinical Practice \\ McPharlin, $\mathbf{N}^{1^{*}}$; Stewart, H. ${ }^{2}$; Gibbs, D. ${ }^{3}$; Bowen-Salter, H. ${ }^{4} \&$ Boshoff, K. $^{4}$ \\ DOI 10.21913/TAHS.v1i1.1558
}

\begin{abstract}
Aim and Background: Occupational Therapists form an essential part of the development and care of infants and families in Neonatal Units (NNU) across the world, however, little is documented about their practice in Australia. This article aims to develop a greater understanding of the role of Occupational Therapy in NNU in Australia. Method: An online questionnaire was developed from a systematic scoping review and piloted by an expert panel. Invitations were sent to thirty Occupational Therapists working in NNUs around Australia. Data analysis included using descriptive statistics and qualitative themes. Results: Twenty two (73\%) of NNU Occupational Therapists responded to the survey, representing all levels of NNU. Results describe the current Occupational Therapy role in Australia as encompassing assessment, intervention, teamwork and a variety of 'other' activities, all of which required advanced knowledge, skills and paediatric experience, and involved ongoing high-level professional development. Occupational Therapy has a unique role to play in

1 Women's and Children's Hospital, Adelaide, Australia

2 Occupational Therapy, Catholic University, Queensland, Australia

3 Clinical Research for Neonates and Children, Children's Research, Barts Health, NHS Trust, Royal London Hospital, London, UK

4 International Centre for Allied Health Evidence, Allied Health and Human Performance, University of South Australia, Adelaide, Australia

1*Correspondence: nancymcpharlin@icloud.com
\end{abstract}


The Allied Health Scholar Vol. 1, No. 1 (2020)

neonatal units. Future development of the role is reliant upon good advocacy and promotion, specialised training opportunities, prospects for networking, support and mentorship for the many Therapists working part-time, and ongoing research. The Australian Occupational Therapy NNU role compares similarly with those cited internationally (UK \& US). The development of guidelines for evidence based clinical practice of neonatal Occupational Therapy in Australia was highly sought by clinicians. The adoption of the 'Occupational Therapy in neonatal services and early intervention: practice guideline' (RCOT 2017) is proposed until the emergence of specific Australian guidelines.

Keywords: Neonatal care, Occupational Therapy, Guidelines

\section{Introduction}

In Neonatal Units, or NNU, Occupational Therapists facilitate the emergence of the Occupational roles of infants and parents, to enhance development and quality of life (AOTA, 2018; RCOT, 2017). They incorporate the influence of the biological, developmental and psychosocial aspects of humans, expressed through meaningful occupations (AOTA, 2018). This perspective provides a unique and valuable addition to the Neonatal Unit, (AOTA, 2018; Nightlinger, 2011) and requires a high level of education, skills and ongoing professional development (Craig \& Smith, 2020).

The rate of premature birth is $10 \%$ world-wide and increasing (Althabe et al., 2010). A concurrent decreased mortality rate of premature infants has focused attention on improving the morbidity of this group (Marlow, 2015) including the quality of NNU care and follow-up. Infants in NNU often face medical complications and developmental issues (Behrman \& Butler, 2007; Kuban et al., 2016; Mouradian et al., 2000). The NNU environment provides life-saving care, however it can bring about negative experiences such as sensory overload and separation from parents (Als, 2009; Altimier, 2013; Smith et al., 
2011). The responses of the sensitive preterm brain to such environmental stressors can change infant brain structure and function (Smith et al., 2011). In addition, premature and sick infants can experience complications including neurological, respiratory, intestinal, immunological, cardiovascular, orthopaedic, genetic and sensory difficulties (Behrman \& Butler, 2007). These include high rates of neurological injury (such as intraventricular haemorrhage and periventricular leukomalacia) which are associated with neurodevelopmental difficulties (Hack \& Taylor, 2000) and are usually more significant for very low birth weight infants (less than 1,500 grams) (Marlow, 2015).

The long term outcomes of extremely preterm infants followed by the ELGAN Study (Kuban et al., 2016; Synnes et al., 2016) identified neurologic (including motor), cognitive and behavioural (including autism spectrum disorder) difficulties. Specific neurodevelopmental issues linked to prematurity include attention deficit hyperactivity disorder, learning disabilities, visual perceptual and visual-motor problems, executive functioning deficits (Behrman \& Butler, 2007) and sensory processing issues (Crozier et al., 2016; Mitchell et al., 2015). These significant long-term ramifications highlight the importance of early intervention to ameliorate long term impacts and include neuroprotective developmental care strategies and therapeutic interventions in NNU (Altimier \& Phillips, 2013; AOTA, 2018; Nightlinger, 2011; RCOT, 2017). Evidence based practices such as individualised developmental care, and allied health team interventions, including Occupational Therapy, have become an integral part of NNU practice (Allinson et al., 2017).

Developmental care practices in NNU continue to evolve and result in improvement in maternal and infant outcomes post NNU (Lipner \& Huron, 2018). These include the Newborn Individualized Developmental Care and Assessment Program (NIDCAP)(Als, 2009), Family Nurture Intervention (Welch et al., 2012), Family Integrated Care (Lee et al., 
The Allied Health Scholar Vol. 1, No. 1 (2020)

2017), and the Mother Infant Transaction Programme (Rauh et al., 1990), Family Integrated Care, (Bangerjee et al., 2018). The Neonatal Integrative Developmental Care Model (Altimier \& Phillips, 2013) provides an example of a neuroprotective developmental care model which can integrate core Occupational Therapy elements such as facilitating infant and parent co-occupations (partnering with families, positioning and handling, minimising pain, optimizing nutrition, protecting skin and sleep) and environmental adaptation (modifying touch, sound, light, smell, and taste) to enhance the healing environment (Altimier \& Philips, 2013).

Research investigating neonatal Occupational Therapy practice has found that developmental interventions resulted in gains in early cognitive development of infants and preschool children (Clark and Schlabach, 2013) and that Occupational Therapy based neuromotor intervention with premature infants lead to beneficial growth outcomes (Aucott et al., 2002). Therefore, understanding the nature and extent of this role in Australia is essential.

Practice guidelines for neonatal Occupational Therapists are provided by the Royal College of Occupational Therapy UK (RCOT, 2017) and a detailed description of this role by The American Occupational Therapy Association position paper (AOTA, 2018). While there are no equivalent Australian guidelines for neonatal Occupational Therapists, their need has been acknowledged (Scannell, 2011). Given that in Australia the incidence of premature birth is $8 \%$, and increasing, with $15 \%$ of these babies requiring extra care at birth (Hilder et al., 2014), Occupational Therapy in the NNU in Australia is in increasing demand. However, this has not been reflected in practice, with the survey by Allinson et al. (2017) of neurodevelopmental allied health teams in Australian and New Zealand Neonatal Nurseries, showing a poorer representation of Occupational Therapy, compared to Physiotherapy and 
The Allied Health Scholar Vol. 1, No. 1 (2020)

Speech Pathology. Some Australian NNUs have no Occupational Therapy involvement and require information on current Australian practice to advocate for role initiation and development. Additionally, health funding for allied health Therapists in Australia is at a critical level whereby justification for services is under increasing scrutiny.

In Australia there is a need for informed descriptions of the roles and responsibilities of neonatal Occupational Therapists to advocate for their place in NNUs to government, hospital administration and consumer groups, and to inform the development of Australian practice guidelines. Results could also facilitate teaching, mentoring and networking within this group and guide future research on the efficacy and outcomes of Occupational Therapy in NNU. As such, this paper reports on the first extensive survey of the role of Occupational Therapy in NNU in Australia and compares this with international clinical practice guidelines before recommending a pathway for Australian guideline development.

\section{Design}

This study was conducted as part of an Honours degree and involved an online survey (Survey Monkey) of Australian NNU Occupational Therapists to capture both descriptive and numeric data (Terwee et al., 2006) about their activities involved in the role of Occupational Therapy in NNU. The choice of a survey enabled the collection of a large amount of data over a range of topics from therapists directly involved in this practice domain (Couper, Traugott \& Lamias, 2001). Password protected on-line distribution gave easy access to therapists, at minimal cost (Couper, Traugott \& Lamias, 2001).

\section{Survey Development}

Quality criteria outlined in Terwee et al. (2006) were used to guide survey development. The survey was designed to answer the question "What is the role of 
The Allied Health Scholar Vol. 1, No. 1 (2020)

Occupational Therapy in NNU in Australia?" To develop an accurate survey tool, a scoping review (involving a systematic search following PRISMA protocols, as in Moher et al., 2009) aimed to explore, condense and describe the role of Occupational Therapy in NNUs. Literature from 2000-2017 was reviewed, with all types of study design included and only English texts. Of the 654 potentially relevant documents, 27 articles were included.

Data extraction from the scoping review informed the development of the survey questions. This covered information about assessments and interventions used by neonatal Occupational Therapists, teamwork, education and skills, gaps in research, and 'other' aspects of the role. The results were synthesised into 52 survey questions following these same themes and added organisational information plus infant and family follow-up (for example questions relating to assessments used by Occupational Therapists in NNU included: 'Which formal and informal assessments do you use in NNU (from the list of 42 formal and 10 informal assessments supplied)?', 'Have you developed your own NNU Assessments?', 'Do you carry out parent assessments?', 'Which aspects of the NNU environment do you assess?', 'Which Assessments would you like training in?'). The author's knowledge and clinical experience, and correspondence with expert authors were also integrated into question formulation.

Survey development theory (Couper, Traugott \& Lamias, 2001) suggested utilising open and closed questions designed for the collection of statistical and descriptive data (DePoy \& Gitlin, 2016), and incorporated multiple choice answers and some 'comments' sections. In order to ensure the appropriateness of content of the questionnaire, an expert panel provided feedback and the questionnaire was trialled. Feedback led to modification of some content including relevance to current practice, readability and useability, formatting, specificity of questions, clarification of Australia specific terminology, and choice of 
The Allied Health Scholar Vol. 1, No. 1 (2020)

question type (Terwee et al., 2006). Expert therapists' feedback deemed the survey a comprehensive capture of the neonatal Occupational Therapy role.

Ethics approval for the study was gained from the Human Research Ethics Committee University of South Australia, and was undertaken following the University of South Australia Framework for the Responsible Conduct of Research. No identifying information was collected. Confidentiality of information gathered from the survey was respected in accordance with the NHMRC ethical code of practice. Completion and return of the questionnaire by participants, indicated consent to participate. An information sheet for participants was sent via email to describe the purpose and scope of the study and to distribute the questionnaire via a Web-Link. Email was also used for follow up. Whilst it was acknowledged that complete confidentiality of electronic data could not be assured, the identity of therapists and their hospitals was non-identifiable. Individual patient data was not collected. Data was stored according to University ethics protocol.

\section{Data Collection and Analysis}

The survey inclusion criteria required participants to be Occupational Therapists working in Australian NNU. The Email addresses for 30 Occupational Therapists working in NNU in Australia were gathered from a previous benchmarking study of 9 therapists (Lyndall Franklin, Occupational therapist, Neonatal Critical Care Unit, Mater Mothers Hospitals, Brisbane, personal communication 2016), websites of Australian hospitals, and telephone calls to NNUs (Terwee et al., 2006).

Data was collected from August 2017. Information collected was not identifiable. The quantifiable data was extracted to an Excel spreadsheet for analysis. Descriptive statistics, including frequencies and percentages, were calculated. 
The Allied Health Scholar Vol. 1, No. 1 (2020)

Qualitative data included descriptive information relating to the Occupational Therapy role in NNU, for example, activities involved in Occupational Therapy practice in NNU, roles of allied health team members and their overlap with Occupational Therapy, report writing, work history, training, ongoing professional development, research, follow-up clinics and treatments, and other activities involved in the NNU role of Occupational Therapy (DePoy and Gitlin 2015). Thematic analysis was utilised for qualitative data conducted by the first author, then verified and agreed by the research team (DePoy \& Gitlin, 2015), and the results were reported in a descriptive format.

\section{Results}

The survey response rate of $73 \%$ ( 22 of 30 participants) provided data on the Occupational Therapy role presented in two categories: 'Participant and organisational demographics'(Table 1) and 'Assessments and interventions'(Table 2) used by neonatal Occupational Therapists. Also reported were 'teamwork' and 'other' activities associated with the role. Therapists were not required to answer all questions, with total responses ranging from $n=17$ to $n=22$.

Survey data which focused on NNU Occupational Therapy role description was selected for discussion in this article, in order to compare the Australian role with international practice and to discuss the need for professional clinical guidelines.

\section{Participant and Organisational Demographics}

NNUs were located in large cities on the east coast of Australia congruent with the population distribution across the country. The majority of therapists, $(n=9,43 \%)$ were considerably experienced with more than 20 years' practice in Occupational Therapy, whereas the majority of Occupational Therapists spent 2-5 years practicing in NNU ( $n=11$, 
The Allied Health Scholar Vol. 1, No. 1 (2020)

$55 \%)$. The title 'Occupational therapist' was used by nearly all respondents ( 20 of $22,95 \%)$, with four others referred to as either 'neonatal', 'neurodevelopmental' or 'developmental' therapist.

Table 1 Descriptive Characteristics of Neonatal Occupational Therapists and the NNU settings

\begin{tabular}{|c|c|c|}
\hline & Number & Percentage \\
\hline \multicolumn{3}{|l|}{ OTs Characteristics } \\
\hline \multicolumn{3}{|c|}{ Years of Practice as an OT } \\
\hline $0-5$ & 1 & $5 \%$ \\
\hline $5-10$ & 4 & $19 \%$ \\
\hline $10-15$ & 4 & $19 \%$ \\
\hline $15-20$ & 3 & $14 \%$ \\
\hline \multirow[t]{2}{*}{$20+$} & 9 & $43 \%$ \\
\hline & 21 & \\
\hline \multicolumn{3}{|c|}{ Years of Practice in NNU } \\
\hline Less than 2 & 1 & $5 \%$ \\
\hline 2 to 5 & 11 & $55 \%$ \\
\hline 5 to 10 & 4 & $20 \%$ \\
\hline \multirow[t]{2}{*}{$10+$} & 4 & $20 \%$ \\
\hline & 20 & \\
\hline \multicolumn{3}{|l|}{ Level of Education } \\
\hline Batchelor & 10 & $56 \%$ \\
\hline Honours & 1 & $6 \%$ \\
\hline Masters & 1 & $6 \%$ \\
\hline \multirow[t]{2}{*}{ Doctorate } & 6 & $33 \%$ \\
\hline & 18 & \\
\hline \multicolumn{3}{|l|}{ OTs in NNU } \\
\hline \multicolumn{3}{|l|}{ Level } \\
\hline Level 1 & 10 & $50 \%$ \\
\hline Level 2 & 6 & $30 \%$ \\
\hline Level 3 & 6 & $30 \%$ \\
\hline Level 4 & 4 & $20 \%$ \\
\hline Level 5 & 6 & $30 \%$ \\
\hline Level 6 & 9 & $45 \%$ \\
\hline \multicolumn{3}{|l|}{ NNU Characteristics } \\
\hline \multicolumn{3}{|l|}{ State of NNU } \\
\hline New South Wales & 9 & $31 \%$ \\
\hline Northern Territory & 1 & $3 \%$ \\
\hline Queensland & 8 & $28 \%$ \\
\hline South Australia & 3 & $10 \%$ \\
\hline Tasmania & 1 & $3 \%$ \\
\hline Victoria & 5 & $17 \%$ \\
\hline Western Australia & 2 & $7 \%$ \\
\hline Total & 29 & $100 \%$ \\
\hline \multicolumn{3}{|l|}{ Number of Cots } \\
\hline NICU & & 5 to 79 , average 37 \\
\hline SCBU & & 6 to 50 , average 24 \\
\hline
\end{tabular}


Occupational Therapists in Australian NNUs were funded for an average of less than 2 days per week. In addition, over $50 \%$ of surveyed therapists worked one day or less per week. Only one therapist worked five days per week. Therapists commented on the limitations of such part-time involvement (e.g. that they were limited to practice with a specific age/weight or diagnostic group of infants) with some therapists only able to respond to urgent referrals. Funding for NNU Occupational Therapy was a common concern for respondents, with over 50\% reporting that their work was funded from the general Occupational Therapy department budget (ie with no specific allocation of funding for NNU work). Therapists recommended a more extensive and consistent presence of Occupational Therapists in NNUs to assist with role establishment and development.

Occupational Therapists worked across Levels 1-6 of NNUs (where Level 1 contains the least fragile infants, to Level 6 with the most fragile). Most Occupational therapists $(\mathrm{n}=19,86 \%)$ cared for infants of all age brackets:

- extremely preterm (i.e. $<28$ weeks, $n=14,74 \%$ )

- $\quad$ very preterm (i.e. 28 to $<32$ weeks, $n=13,68 \%$ )

- moderate to late preterm (i.e. 32 to $<37$ weeks, $n=16,84 \%$ )

- older than 37 weeks $(\mathrm{n}=12,63 \%)$.

Therapists $(n=19,86 \%)$ care for infants in all weight categories: 2,500 grams $(n=12,63 \%)$;

low birth weight $<2,500$ grams $(n=13,68 \%)$; very low birth weight $<1,500$ grams $(n=14$, 74\%); extremely low birth weight $<1,000$ grams $(\mathrm{n}=14,74 \%)$. 
The Allied Health Scholar Vol. 1, No. 1 (2020)

Table 2 Assessments and Interventions utilised by Australian neonatal occupational therapists

\begin{tabular}{|c|c|c|}
\hline & $\begin{array}{c}\text { Number of } \\
\text { Respondents } \\
\text { Total } n=17 \\
20,20\end{array}$ & $\begin{array}{c}\text { Percentage of } \\
\text { Respondents } \\
\%\end{array}$ \\
\hline General Movements Prechtl (GMs) & 14 & $82 \%$ \\
\hline Bayley Scale of Infant Toddler Development Version 3 (BSITD-3) & 11 & $65 \%$ \\
\hline Dubowitz Neurological Assessment of Preterm \& Full-Term Infants (Dubowitz) & 8 & $47 \%$ \\
\hline Hammersmith Neonatal Neurobehavioral Examination (NNE) & 7 & $41 \%$ \\
\hline Test of Infant Motor Performance (TIMP) & 6 & $35 \%$ \\
\hline Peabody Developmental Motor Scale 2 (PDMS-2) & 4 & $24 \%$ \\
\hline Brazelton Neonatal Assessment Scale (NBAS) & 3 & $18 \%$ \\
\hline NICU Network Neurobehavioral Scale (NNNS) & 3 & $18 \%$ \\
\hline Alberta Infant Motor Scales (AIMS) & 2 & $12 \%$ \\
\hline Infant Position Assessment Tool (IPAT) & 1 & $6 \%$ \\
\hline Motor Assessment of Infants (MAI) & 1 & $6 \%$ \\
\hline Neuro Sensory Motor Development Assessment (NSMDA) & 1 & $6 \%$ \\
\hline Newborn Individualised Developmental Care \& Assessment Program (NIDCAP) & 1 & $6 \%$ \\
\hline Supporting Oral Feeding In Fragile Infants (SOFFI) & 1 & $6 \%$ \\
\hline \multicolumn{3}{|l|}{ Informal Assessments } \\
\hline Developmental Care & 17 & $85 \%$ \\
\hline Neurobehavioral & 16 & $80 \%$ \\
\hline Musculoskeletal & 16 & $80 \%$ \\
\hline Environmental Modifications & 16 & $80 \%$ \\
\hline Neurodevelopmental & 14 & $70 \%$ \\
\hline Sensory and Sensory Loss & 11 & $55 \%$ \\
\hline Family & 11 & $55 \%$ \\
\hline Head Shape & 9 & $45 \%$ \\
\hline Infant Mental Heath & 8 & $40 \%$ \\
\hline Oromotor and feeding & 7 & $35 \%$ \\
\hline Pain & 3 & $15 \%$ \\
\hline \multicolumn{3}{|l|}{ Interventions } \\
\hline Positioning and Handling & 19 & $95 \%$ \\
\hline Developmental Care & 18 & $90 \%$ \\
\hline Play / Interaction & 18 & $90 \%$ \\
\hline Infant States and Cues & 17 & $85 \%$ \\
\hline Environmental Modification & 17 & $85 \%$ \\
\hline Parent Engagement and Support & 16 & $80 \%$ \\
\hline Neurodevelopmental & 15 & $75 \%$ \\
\hline Positive Touch and Massage & 15 & $75 \%$ \\
\hline Daily Care Routines & 14 & $70 \%$ \\
\hline Skin to Skin Kangaroo Care & 13 & $65 \%$ \\
\hline Orthopaedic & 13 & $65 \%$ \\
\hline Parent Education Groups & 11 & $55 \%$ \\
\hline Sensory and Sensory Loss & 9 & $45 \%$ \\
\hline Neuromotor & 8 & $40 \%$ \\
\hline Infant Mental Health & 7 & $35 \%$ \\
\hline Oro-motor and Feeding & 6 & $30 \%$ \\
\hline Pain Management & 4 & $20 \%$ \\
\hline
\end{tabular}


The Allied Health Scholar Vol. 1, No. 1 (2020)

The percentage of therapists involved in various diagnostic groups included prematurity $90 \%$, central nervous system dysfunction $85 \%$, genetic syndromes $95 \%$, respiratory $70 \%$, cardiac 60\%, orthopaedic (including splinting) 75\%, surgical 55\%, sensory impairment (hearing and/or vision) $75 \%$, and parent/caregiver diagnostic groups 55\%.

Australian neonatal intensive care unit (NICU) cot numbers varied from 5-79 (averaging 37 cots) and special care baby units (SCBUs) ranged from 6-32 cots (averaging 22 cots). Therapists reported a range of care models being used in NNUs, including developmental care $(n=15,78 \%)$, family focused care $(n=10,53 \%)$, biomedical $(n=6,32 \%)$ and preventative care $(n=2,11 \%)$, with therapists commenting that these care models were adopted to varying degrees and combinations within each unit.

The referral of infants for Occupational Therapy was not necessarily obligatory in all settings. Referrals were described as either 'Open' (i.e. automatic referral to Occupational Therapy, $n=12,57 \%$ ), 'Individualised' (i.e. one referral specific to an infant, $n=12$ therapists, $57 \%$ ), or 'Other' including a mix of both referral styles ( $\mathrm{n}=10$ therapists, $48 \%)$. Two therapists described time constraints limiting the acceptance of referrals.

\section{Assessments}

The assessments used in the survey questions were consistent with findings of the scoping review. Assessments overlapped in their content and focus, for example an assessment of developmental care may incorporate aspects of infant development, interaction with others, sensory concerns, pain and the NNU environment. Assessments used by Australian neonatal Occupational Therapists (Table 2) showed that formal (standardised) assessments commonly focused on neuro-behaviour and neurodevelopment, whereas informal assessments covered a broad range of practice areas. Some Occupational Therapists 
The Allied Health Scholar Vol. 1, No. 1 (2020)

had developed their own assessments ( 3 of 21 respondents, 14\%), and 17 therapists requested training in formal assessments.

\section{Interventions}

Occupational Therapists commented that part-time work in NNUs limited the range of activities they were able to engage in. When therapy hours were particularly limited a prescribed range of duties such as splinting, or discharge planning were offered. In others $(n=4)$ fulltime practice allowed for a wide scope of practice incorporating developmental care, assessments and interventions. The range of interventions used by respondents ( 20 of 22, 91\%) (see Table 2) show that positioning and handling occurred most frequently, and pain management least frequently. Feeding interventions included implementing nonnutritive suck, pre-feeding groups and support during the establishment of feeding.

Parent education group topics included infant massage, positioning and handling, infant behaviour and cues, sensory environments, sleep, play and sensory processing, and appropriate toy and equipment selection. Amongst these groups five therapists (45\%) conducted pre-discharge groups, with three (27\%) conducting post-discharge groups. Discharge planning incorporated: provision of a home program, equipment and technology selection, supply and modification, activities of daily living and home visiting.

\section{Teamwork and role delineation in $N N U$}

Respondents (20 of 22, 91\%) described their NNU allied health team workers in order of prevalence: physiotherapist $(n=20,100 \%)$, Speech pathologist $(n=18,90 \%)$, social worker $(n=18,90 \%)$, dietician $(n=12,60 \%)$ and psychologist $(n=4,20 \%)$. Other team members included: music therapist, health practitioner, indigenous health practitioner and liaison officer and lactation consultant. Respondents also identified the areas in which Occupational Therapy overlapped with Physiotherapy $(n=19)$ and Speech Pathology $(n=20)$ practice. Data 
The Allied Health Scholar Vol. 1, No. 1 (2020)

and discussion of these aspects of the Occupational Therapy role in NNU can be found in McPharlin (2018).

NNU team consultations incorporated a range of scenarios such as: discharge planning $(n=16,84 \%)$, case discussion $(n=14,76 \%)$, ward rounds $(n=13,68 \%)$, ethical discussions $(n=5,26 \%)$, and diagnostic discussions $(n=4,21 \%)$. Therapists noted funding constraints restricted their involvement in these activities. Some therapists reported difficulties with multidisciplinary team dynamics and breaking into the culture of NNU. Therapists addressed these issues via networking, and support with their Occupational Therapy colleagues, and linked these issues with the need for a clear role description and Australian evidence-based guidelines for practice.

\section{'Other' activities in the OT role in $N N U$}

Other activities in the neonatal Occupational Therapy role were reported as quality assurance, research, committees and teaching of staff and students. Respondents, 21(95\%), reported that administrative duties took from 0 to 4 hours per week. Promotion and development of neurodevelopmental care, the review and recommendation of NNU equipment, outreach to rural areas and contributing to state-wide policy documents, were other activities described. Respondents reported the increasing use of technology by therapists, citing filming, photography, and the development and use of mobile phone Apps, videoconferencing and electronic record keeping. An over-riding theme from therapists' responses was the need advocate for the emerging Occupational Therapy role in NNU in Australia.

\section{The need for Australian-specific guidelines for practice}

The development of Australian guidelines for clinical practice of Occupational Therapy in NNU was strongly supported by $83 \%$ of surveyed therapists to describe their role 
The Allied Health Scholar Vol. 1, No. 1 (2020)

and support applications for the development and expansion of neonatal Occupational Therapy positions.

\section{Discussion}

In discussing the aspects of the role of Occupational Therapy in NNU, the breadth and complexity of practice areas became evident, highlighting the necessity of ongoing training for these therapists.

Australian neonatal Occupational Therapy staffing levels reveal the small foothold therapists have in this specialised area of practice. Over $50 \%$ of surveyed therapists worked one day or less per week. These therapists describe their work as 'an occasional single referral' and were at the forefront of establishing a more significant service in their NNU. Further data shows $14 \%(n=3)$ work 2 days/week, 5\%(n=1) work 3 days/week, 14\%(n=3) work 4 days/week, and 5\%(n=1) work 5 days per week. On average Australian Occupational Therapists worked less than 2 days per week in NNUs. Data was not collected on days worked per week related to the number of cots per NNU and therefore cannot be compared to recent literature. For example, in the US, Craig and Smith (2020) proposed a formula to calculate the full time equivalent neonatal therapy hours (including Occupational Therapists, Physiotherapists and Speech Pathologists) for various levels of NNU, within an integrated model of care. The UK Occupational Therapy staffing on Neonatal Units guidelines (RCOT, 2018) recommended minimum staffing levels of 0.05-0.1 FTE per intensive care cot, 0.0250.05 FTE per high dependency cot, and similarly 0.025-0.05 FTE per special care cot, acknowledging that this would vary according to the availability of other neonatal therapists (Physiotherapists and Speech Pathologists) working with this population. Australian neonatal Occupational Therapists could utilise the recommendations of RCOT (2018) and Craig and Smith (2020) to advocate for research, establishment and development this Australian role. 
The Allied Health Scholar Vol. 1, No. 1 (2020)

The assessment of NNU parents/carers (by 55\% of respondents) will be an ongoing focus with research indicating significant levels of stress in parents pre- and post- admission to NNU (Lee \& O'Brien, 2017). The UK Guidelines (RCOT, 2017) recommend interventions which alleviate parental stress, suggesting that programs which enhance parental involvement in infant care may provide benefits for both parent and child in NNU and beyond discharge (Lee \& O'Brien, 2017). Further, $80 \%$ of Australian therapists were involved in parent engagement and support, to encourage the facilitation of parenting cooccupations in NNU (Barbosa, 2013).

Australian Occupational Therapists were engaged in diverse interventions in NNUs (Table 2.). The main difference found between Australian and American Occupational Therapy roles in NNU, was involvement in infant oro-motor and feeding development. Ross et al. (2017) identified the Occupational therapist as most frequently engaged in feeding interventions in the US, whereas only $33 \%$ of Australian Occupational Therapists were involved in feeding and identified Australian Speech Pathologists and NNU nurses as usually fulfilling this role. Such trends in NNU practice were described as having a historical background.

Australian neonatal Occupational Therapists worked primarily in multidisciplinary therapy teams due to the complexity of NNU infants and the common incidence of neurobehavioural impairment of infants born before 32 weeks gestation. This was supported by Ross et al. (2017) and Barbosa (2013), who encouraged teamwork whilst retaining the unique character of each therapy in NNU practice. Challenges related to teamwork (i.e. role overlap and delineation, and difficulty breaking into the NNU culture), were mentioned by survey participants. These challenges were supported by Hunter, Lee and Altimier, (2014) and research was suggested into this phenomenon (Carretto et al., 2000). Part-time work and role 
The Allied Health Scholar Vol. 1, No. 1 (2020)

overlap with multidisciplinary colleagues, led Australian neonatal Occupational Therapists to request clear role delineation (Allinson, 2017; Scannell, 2015) including the development of guidelines for Occupational Therapy in NNU for Australia. Survey respondents reported (see Table 2) a high frequency of use of formal and informal assessments of neurobehaviour and neurodevelopment (80-85\%). and interventions in positioning and handling, developmental care, play and interaction, infant states and cues, environmental modification, parent engagement and support, neurodevelopment, positive touch and massage, daily routines, skin to skin, orthopaedic and sensory (from 95-45\% respectively). Conversely, fewer respondents reported utilising infant metal health, pain and feeding assessments and interventions $(35,30$ $20 \%$ respectively), possibly due to a lack of training opportunities therapists requested training in these areas. Other NNU team members may have worked in these areas. The lack of funding and opportunity for training was reported as a barrier to neonatal Occupational Therapy development in Australia. Overall, the survey findings describe the role of neonatal Occupational Therapy in Australia as being in an evolutionary phase, requiring significant expansion, development and research.

\section{Practice Implications}

Firstly, the results confirm the complexities of the neonatal Occupational Therapy role which require a high level of knowledge, skills and experience, together with ongoing professional development and support (AOTA 2018). Australian neonatal Occupational Therapists reported utilising both UK guidelines (RCOT, 2017) and the American position paper (AOTA, 2018) to inform their practice, as well as research publications and other training and conference opportunities. Online courses from NANT (National Association of Neonatal Therapists, US) and other online training providers are also used. The NANT annual conference provides an annual critical review of recent publications relevant to neonatal therapy is a popular source of professional development material. The development 
The Allied Health Scholar Vol. 1, No. 1 (2020)

of an Australian Neonatal Occupational Therapy Special Interest Group was supported by surveyed therapists. Alternatively, or in conjunction, Australia could adopt a multidisciplinary neonatal therapy interest group.

Secondly, the results recommend the development of evidence based Australian guidelines for the practice of Occupational Therapy in NNU. As a result, the authors undertook a comparison of the survey results with the UK guidelines' descriptive categories (RCOT, 2017), see Table 3. The table shows that Australian therapists engage in many activities identified as 'Neonatal OT'.

\section{Guideline adoption and implementation}

A comparison of Australian neonatal Occupational Therapy practice areas with the UK guideline recommendations (RCOT, 2017) shows a high correlation in most practice domains (Table 3.). Therefore, it could be proposed that until specific Australian guidelines evolve, adopting the UK guidelines (RCOT, 2017) with its supporting documents, represents a valid way to develop this practice. The American position paper (AOTA, 2018) provides additional information to support this process. For neonatal Occupational Therapists working in isolation both in Australia and abroad, the adoption of these UK and American resources could also be beneficial in guiding emerging clinical practice.

Phase 1:

The Australian survey respondents reported a high level of engagement in advocacy for role development. This could be enhanced by enabling opportunities to promote the UK practice guideline and evidence-based recommendations with neonatal multidisciplinary colleagues and in NNU and professional meetings. The RCOT (2017) continuing professional development power-point resource may support these discussions and can be tailored for local use (RCOT, 2017). 
The Allied Health Scholar Vol. 1, No. 1 (2020)

Table 3 Comparison of UK Guidelines with Australian neonatal occupational therapy

\begin{tabular}{|c|c|c|}
\hline UK Guidelines RCOT 2017 (*) & Australian Survey Results 2018 & $\begin{array}{c}\% \text { of therapists } \\
\text { performing these } \\
\text { activities }\end{array}$ \\
\hline \multirow{2}{*}{ Neuro-behavioural } & Neuro-behavioural assessments & $80 \%$ \\
\hline & Neuro-behavioural interventions & $80 \%$ \\
\hline \multirow{7}{*}{ Neurodevelopmental } & Infant states \& cues & $95 \%$ \\
\hline & Neurodevelopmental interventions & $95 \%$ \\
\hline & Play/interaction (see also Developmental Care) & $90 \%$ \\
\hline & Neurodevelopmental assessments & $82 \%$ \\
\hline & Musculoskeletal assessment & $80 \%$ \\
\hline & Head shape intervention & $45 \%$ \\
\hline & Musculoskeletal intervention & $45 \%$ \\
\hline \multirow{6}{*}{ Developmental Care } & Developmentally supportive care & $90 \%$ \\
\hline & Environmental modification & $85 \%$ \\
\hline & Environmental assessment & $80 \%$ \\
\hline & Parent engagement \& support & $80 \%$ \\
\hline & Sensory assessment & $45 \%$ \\
\hline & Sensory intervention & $45 \%$ \\
\hline \multirow{7}{*}{ Pain } & Parent engagement and support & $80 \%$ \\
\hline & Positive touch and massage & $75 \%$ \\
\hline & Skin to Skin Care & $65 \%$ \\
\hline & Infant mental health assessment & $40 \%$ \\
\hline & Infant mental health intervention & $35 \%$ \\
\hline & Pain management & $20 \%$ \\
\hline & Pain assessment & $15 \%$ \\
\hline \multirow{3}{*}{ Positioning } & Developmental positioning & $95 \%$ \\
\hline & Individualised positioning & $95 \%$ \\
\hline & Infant Positioning tool & $6 \%$ \\
\hline \multirow{2}{*}{ Infant Feeding } & Feeding assessment & $35 \%$ \\
\hline & Feeding intervention & $30 \%$ \\
\hline \multirow{6}{*}{$\begin{array}{l}\text { Parent Engagement } \\
\text { Build a positive therapeutic collaboration with } \\
\text { parents }\end{array}$} & Play and interaction & $95 \%$ \\
\hline & Developmental care & $90 \%$ \\
\hline & Parent engagement and support & $80 \%$ \\
\hline & Daily care routines & $70 \%$ \\
\hline & Skin to Skin Care & $65 \%$ \\
\hline & Infant Feeding & $30 \%$ \\
\hline \multirow{3}{*}{ Parent Support } & Environmental modifications & $80 \%$ \\
\hline & Parent engagement and support & $80 \%$ \\
\hline & Daily routines & $70 \%$ \\
\hline
\end{tabular}

* Royal College of Occupational Therapists (2017) Occupational therapy in neonatal services and early intervention: practice guideline. London: RCOT. 
The Allied Health Scholar Vol. 1, No. 1 (2020)

Phase 2:

Auditing current neonatal Occupational Therapy service provision against the UK guidelines would enable consideration of areas of embedded and emerging practice, and relevance in the local context. RCOT (2017) included an audit form that is available for download that could be implemented and form a basis for developing an action plan for service development. There is also an ongoing focus for Australian neonatal Occupational Therapists to engage in research, and publication of research findings is emerging.

Phase 3:

The provision of ongoing reporting of service activity and clinical outcomes are an important element in sustaining and developing neonatal Occupational therapist services. Activities recommended in the RCOT (2017) guideline suggest a multifactorial approach that may also be relevant Australian context. These include:

- Routine reporting of infant and family clinical outcomes using standardised assessments and measures.

- Utilise the evidence-based guidelines as supporting evidence for neonatal Occupational Therapy developments as part of business planning and service commissioning cycles.

- Incorporate implementation case studies demonstrating the translation of the guidelines into local practice to enable infants and their families to receive evidencedbased caregiving.

- Integrate service activity reporting with feedback from service users (parents) to demonstrate the difference that neonatal Occupational Therapy involvement is having 
The Allied Health Scholar Vol. 1, No. 1 (2020)

on parent/infant experience, quality of care provided, and service effectiveness RCOT (2017).

\section{Research Implications}

Research into the hours worked in NNU by Occupational Therapists needs to go beyond those collected by this study. Greater specificity such as hours spent at each level of NNU with regards to the number of cots per unit, would enable the number of therapy hours per cot to be calculated which could then be compared to the UK (RCOT 2018) and American (Craig \& Smith 2020) formulae. This would enable advocacy to hospital administration and government policy makers for appropriate staffing levels with internationally recommended standards of care.

Research was also warranted in the areas of:

- Multidisciplinary team dynamics in NNU

- The role of Occupational Therapy in developmental care

- The introduction of Neonatal Therapy Certification or similar educational requirement for therapists working in NNUs

- Mentoring of therapists training in neonatal therapy

- The development of Occupational Therapy roles in feeding, pain management and infant mental health which were underrepresented in this study

- Stress management and support systems for therapists working in NNUs

- The development of guidelines for Occupational Therapy clinical practice in Australian NNUs. 
The Allied Health Scholar Vol. 1, No. 1 (2020)

\section{Barriers and Enablers}

While the need and potential for Australian neonatal Occupational Therapy was strongly reported in the survey, it was also indicated that limited financial resources restricted the initiation and expansion of this service. Whilst over half the survey respondents worked one day per week or less in NNU they would be in a prime situation for advocacy and yet also challenged by how best to utilize their limited time. NNU team dynamics were sometimes reported as being a barrier to role establishment. Extensive education and training for neonatal Occupational Therapy including mentoring during early practice was described as a role enabler, however financial and time restraints limited access to this education. Advocacy using the evidence-based UK guidelines (RCOT, 2017), the American role description, and increasing clinical evidence of the positive impact of neonatal Occupational therapy, all provided a constructive pathway for role development. Australian paediatric Occupational Therapists often covered a mixed caseload incorporating a number of practice areas (eg neonatal, orthopaedic, neurology, trauma and burns). Professional development across more than one specialized area of paediatric Occupational therapy was reported as a barrier in limiting training in neonatal therapy.

\section{Conclusion}

In conclusion, the survey results provided a description of the Occupational Therapy role in NNUs in Australia, and illustrated that the role was evolving, with most NNU Occupational Therapists working less than two days per week. Therefore strong advocacy and action to establish the role further was required, including consideration for the professional development and support of therapists. Whilst unique Australian evidence based clinical practice guidelines would take time to evolve, the adoption of UK guidelines aligned well with the survey findings of current Australian practice and provided a constructive pathway for the future development of neonatal Occupational Therapy. 
The Allied Health Scholar Vol. 1, No. 1 (2020)

\section{Key Points}

- Australian neonatal Occupational Therapy is an advanced practice area requiring highly specialized training, skills and continuing professional development including mentoring.

- This role is in an evolutionary phase, requiring ongoing advocacy, development and research.

- Adoption of the UK guidelines for neonatal Occupational Therapy (RCOT, 2017) in Australia is proposed, whilst Australian guidelines evolve.

- A Special Interest Group to support Australian neonatal Occupational Therapists who often work in isolation, is highly recommended.

Funding: This research received no specific grant from any funding agency in the public, commercial, or not-for-profit sectors.

Conflicts of Interest: There are no conflicts of interest pertaining to this article.

\section{References}

Allinson, L. A., Doyle, L. W., Denehy, L. \& Spittle, A. J. (2017). Survey of neurodevelopmental allied health teams in Australian and New Zealand neonatal Nurseries: Staff profile and standardised neurobehavioral/neurological assessment. Journal of Paediatrics and Child Health, 53, 578-584. https://doi.org/10.1111/jpc.13484

Als, H. (1986). A synactive model of neonatal behavioural organization: framework for the assessment of neurobehavioral development in the premature infant and for support of infants and parents in the neonatal intensive care environment. Physical \& Occupational Therapy in Paediatrics, 6, 3-53. https://doi.org/10.1080/J006v06n03_02 
Als, H. (2009). Newborn individualized developmental care and assessment program: New frontiers for neonatal and perinatal medicine. Journal of Neonatal-Perinatal Medicine, 2, 135-147. https://doi.org/10.3233/NPM-2009-0061

Althabe, F., Howson, C. P., Kinney, M. \& Lawn, J. (2010). World Health Organization. Born too soon: The global action report on preterm birth. https://doi.org/10.1186/1742-4755-10$\underline{\text { S1-S1 }}$

Altimier, L. \& Phillips, R. M. (2013). The neonatal integrative developmental care model: Seven neuroprotective core measures for family-centred developmental care. Newborn and Infant Nursing Reviews, 13(1), 9-22. https://doi.org/10.1053/j.nainr.2012.12.002

American Occupational Therapy Association. (2018). Occupational therapy's role in the neonatal intensive care unit. American Journal of Occupational Therapy, 72 (Suppl. 2), 7212410020. https://doi.org/10.5014/ajot.2018.72S204

Aucott, S., Donohue, P. K., Atkins, E., \& Allen, M. C. (2002). 'Neurodevelopmental Care in the NICU'. Mental Retardation and Developmental Disabilities Research Reviews, 8, 298308. https://doi.org/10.1002/mrdd.10040

Banerjee, J., Aloysius, A., Platonos, K., \& Deierl, A. (2018). Family centred care and family delivered care-What are we talking about? Journal of Neonatal Nursing, 24(1), 8-12. https://doi.org/10.1016/j.jnn.2017.11.004

Barbosa, V. M. (2013). Teamwork in the neonatal intensive care unit. Physical \& Occupational Therapy in Pediatrics, 33(1), 5-26. https://doi.org/10.3109/01942638.2012.729556

Behrman, R.E. \& Butler, A.S. (2007). Mortality and acute complications in preterm infants. In Preterm birth: causes, consequences, and prevention. US National Academies Press. https://www.ncbi.nlm.nih.gov/books/NBK11385/

Caretto, V., Topolski, K. F., Linkous, C. M., Lowman, D. K., \& Murphy, S. M. (2000). Current parent education on infant feeding in the neonatal intensive care unit: The role of the 
The Allied Health Scholar Vol. 1, No. 1 (2020)

Occupational therapist. American Journal of Occupational Therapy, 54(1), 59-64. http://ajot.aota.org/pdfaccess.ashx?url=/data/journals/ajot/930127/

Clark, G. J. F. \& Schlabach, T. L. ( 2013). 'Systematic review of Occupational therapy interventions to improve cognitive development in children ages birth-5 years', American Journal of Occupational Therapy, 67( 4), 425-430.

https://doi.org/10.5014/ajot.2013.006163

Collins, D, (2003). 'Pretesting survey instruments: an overview of cognitive methods', Quality of Life Research, 12(3), 229-238. https://doi.org/10.1023/A:1023254226592

Couper, M. P., Traugott, M. W., \& Lamias, M. J. (2001). Web survey design and administration. Public Opinion Quarterly, 65(2), 230-253. https://doi.org/10.1086/322199

Craig, J. W., \& Smith, C. R. (2020). Risk-adjusted/neuroprotective care services in the NICU: The elemental role of the neonatal therapist (OT, PT, SLP). Journal of Perinatology, 40,549-559. http://doi.org/10.1038/s41372-020-0597-1

Crookes, P., \& Davies, S. (Eds.) (2004). Research into practice: Essential skills for reading and applying research in nursing and health care. UK: Baillière Tindall.

Crozier, S. C., Goodson, J. Z., Mackay, M. L., Synnes, A. R., Grunau, R. E., Miller, S. P., \& Zwicker, J. G. (2016). Sensory processing patterns in children born very preterm. American Journal of Occupational Therapy, 70(1), 1-

7. https://doi.org/10.5014/ajot.2016.018747

DePoy, E., \& Gitlin, L. N. (2015). Introduction to research-e-book: Understanding and applying multiple strategies. US Elsevier Health Sciences.

Hack, M. \& Taylor, H. G. (2000). Perinatal brain injury in preterm infants and later neurobehavioral function. JAMA, 284(15), 1973-1974. http://doi:10.1001/jama.284.15.1973 
The Allied Health Scholar Vol. 1, No. 1 (2020)

Hilder, L., Zhichao, Z., Parker, M., Jahan, S. \& Chambers, G. (2014). Australia's mothers and babies 2012. Retrieved from. https: APO Analysis and Policy Observatory

Hutchon, B., Gibbs, D., Harniess, P., Jary, S., Crossley, S. L., Moffat, J. V. \& Basu, A. P. (2019). Early intervention programmes for infants at high risk of atypical neurodevelopmental outcome. Developmental Medicine \& Child Neurology, 61(12), 1362-1367. http://doi.org/10.1111/dmcn.14187

Hunter, J., Lee, A. \& Altimier, L. (2014). Neonatal intensive care unit. In J. Case Smith (Ed.), Occupational therapy for children and adolescents (7th Ed., pp. 617-657). Elsevier. https://evolve.elsevier.com/cs/product/9780323169257?role=student

Kuban, K.C., Joseph, R. M., O'Shea, T. M., Allred, E. N., Heeren, T., Douglass, L., Stafstrom, C. E., Jara, H., Frazier, J. A., Hirtz, D. \& Leviton, A. (2016). Girls and boys born before 28 weeks' gestation: Risks of cognitive, behavioral, and neurologic outcomes at age 10 years. The Journal of Pediatrics, 173, 69-75. https://doi.org/10.1016/j.jpeds.2016.02.048

Lee, S. K., \& O'Brien, K. (2017). Family integrated care: Changing the NICU culture to improve whole-family health. Journal of Neonatal Nursing, 24, 1-3 https://doi.org/10.1016/j.jnn.2017.11.003

Lipner, H. S., \& Huron, R. F. (2018). Developmental and interprofessional care of the preterm infant: Neonatal intensive care unit through high-risk infant follow-up. Pediatric Clinics, 65(1), 135-141. https://doi.org/10.1016/j.pcl.2017.08.026

Marlow, N. (2015). Keeping up with outcomes of infants born at extremely low gestational ages. Journal of the American Medical Association, 169(3), 207-208. http://doi:10.1001/jamapediatrics.2014.3362

Mitchell, A. W., Moore, E. M., Roberts, E. J., Hachtel, K. W., \& Brown, M. S. (2015). Sensory processing disorder in children ages birth-3 years born prematurely: A systematic review. 
The Allied Health Scholar Vol. 1, No. 1 (2020)

American Journal of Occupational Therapy, 69(1),1-11. http://doi:10.5014/ajot.2015.013755

Moher, D., Liberati, A., Tetzlaff, J. \& Altman, D.G. (2009). Preferred reporting items for systematic reviews and meta-analyses: The PRISMA statement. Annals of Internal Medicine, 151(4), 264-269. DOI: 10.7326/0003-4819-151-4-200908180-00135

Mouradian, L. E., Als, H., \& Coster, W. J. (2000). Neurobehavioral functioning of healthy preterm infants of varying gestational ages. Journal of Developmental and Behavioral Pediatrics: JDBP, 21(6), 408-416. http://doi.org/10.1097/00004703-200012000-00002

Nightlinger, K. (2011). Developmentally supportive care in the neonatal intensive care unit: An Occupational therapist's role. Neonatal Network, 30(4), 243. http://doi.org/10.1891/0730$\underline{0832.30 .4 .243}$

Rauh, V. A., Nurcombe, B., Achenbach, T., \& Howell, C. (1990). The Mother-Infant Transaction Program: The content and implications of an intervention for the mothers of lowbirthweight infants. Clinics in Perinatology, 17(1), 31-45.

http://doi.org/10.1016/j.infbeh.2008.09.004

Ross, K., Heiny, E., Conner, S., Spener, P., \& Pineda, R. (2017). Occupational therapy, physical therapy and speech-language pathology in the neonatal intensive care unit: Patterns of therapy usage in a level IV NICU. Research in Developmental Disabilities, 64, 108-117. https://doi.org/10.1016/j.ridd.2017.03.009

Royal College of Occupational Therapists. (2017). Occupational Therapy in Neonatal Services and Early Intervention: Practice Guideline. London: RCOT. Retrieved from https://www.rcot.co.uk/practice-resources/rcot-publications/downloads/neonatal-services 
The Allied Health Scholar Vol. 1, No. 1 (2020)

Royal College of Occupational Therapists. (2018). Occupational Therapy Staffing on Neonatal Units. London: RCOT. Retrieved from https://www.rcot.co.uk/practice-resources/rcotpublications/downloads/neonatal-services

Scannell, M. (2011). Finding occupation in a neonatal environment: A review of the literature and current practice. Australian Occupational Therapy Journal, 58, 97http://doi.org/10.1111/j.1440-1630.2011.00938.x

Smith, G. C., Gutovich, J., Smyser, C., Pineda, R., Newnham, C., Tjoeng, T. H. \& Inder, T. (2011). Neonatal intensive care unit stress is associated with brain development in preterm infants. Annals of Neurology, 70(4), 541-549. https://doi.org/10.1002/ana.22545

Synnes, A., Luu, T. M., Moddemann, D., Church, P., Lee, D., Vincer, M. \& Sauve, R. (2016). Determinants of developmental outcomes in a very preterm Canadian cohort. Archives of Disease in Childhood-Fetal and Neonatal Edition. 102(3), F235-234. http://doi.org/10.1136/archdischild-2016-311228

Terwee, C. B., Bot, S. D. M., de Boer, M. R., van der Windt, D. A. W. M., Knol, D. L., et al. (2007). Journal of Clinical Epidemiology, 60(12), 1315-1316. http://doi.org/10.1016/j.jclinepi.2007.06.002

Welch, M. G., Hofer, M. A., Brunelli, S. A., Stark, R. I., Andrews, H. F., Austin, J., \& Myers, M. M.(2012). Family nurture intervention (FNI): methods and treatment protocol of a randomized controlled trial in the NICU. Family nurture intervention (FNI) Trial Group. BMC Pediatrics. 12(14). doi: 10.1186/1471-2431-12-14 